\title{
Mathematical Modelling of Leukemia Treatment
}

\author{
INNA SAMUILIK ${ }^{1}$, FELIX SADYRBAEV ${ }^{1,2}$, \\ ${ }^{1}$ Department of Natural Sciences and Mathematics \\ Daugavpils University \\ Parades street1 \\ LATVIA \\ ${ }^{2}$ Institute of Mathematics and Computer science \\ University of Latvia \\ Rainis boulevard 29 \\ LATVIA
}

Abstract: Leukemia is a cancer that can be treated in a variety of ways: chemotherapy, radiation therapy and stem cell transplant. Recovery rates for this disease are relatively high, the treatment itself has a painful effect on the body and is accompanied by numerous side effects that can persist years after the patient is cured. For this reason, efforts are underway worldwide to develop more selective therapies that will only affect leukemia cells and not healthy cells.

Knowledge of developmental GRN is yet scarce, and it is early for a systematic comparative effort. We consider mathematical model of genetic regulatory networks. This model consists of a nonlinear system of ordinary differential equations. We describe the changes that system undergoes if the entries of the regulatory matrix are perturbed in some way. We discuss, how attractors for highdimensional systems can be constructed, using known attractors of low-dimensional systems. Examples and visualizations are provided.

Key-Words: leukemia, differential equations, attractors

Received: April 20, 2021. Revised: October 1, 2021. Accepted: October 13, 2021. Published: October 23, 2021.

\section{Introduction}

Leukemia ("blood cancer") is a malignant disease of the hematopoietic system. The disease occurs in the bone marrow and then spreads to the blood, lymph nodes, spleen, liver, central nervous system (CNS), and other organs. Leukemia differs from other types of cancer in that it does not form a tumor in any one place. It develops rapidly, more often children and adults under 30 get sick with it. Leukemia is the most common cancer in children. Most often the acute lymphoblastic leukemia occurs in children aged from one to five years. And boys get sick a little more often than girls. The leukemia affects $2-3 \%$ of all cancer patients in the world.

The incidence of leukemia is high in developed countries, in particular among the white population of the United States, Hawaiian Islands, Australia, Europe.
High mortality from leukemia is noted in Eastern Europe: Estonia (male - 7.31; female - 4.24), Latvia (male - 6.59; female - 4.53), Lithuania (male - 7.16; female - 3.99), Poland (male - 6.60; female-3.56).

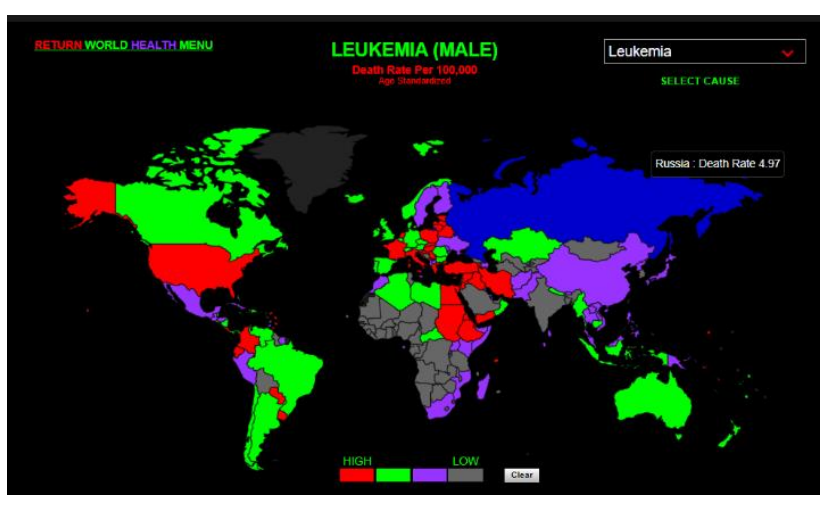

Leukemia (MALE) Death Rate Per 100,000 Age Standardized(https://www.worldlifeexpectancy.com/causeof-death/leukemia/by-country/male) 


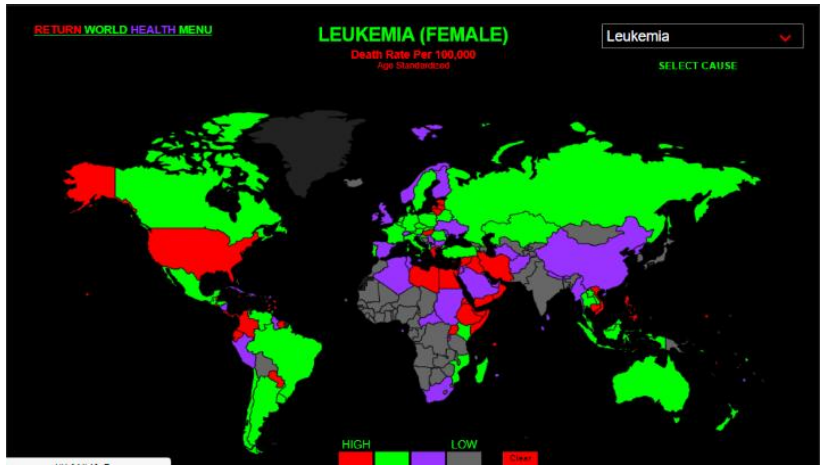

Leukemia (FEMALE) Death Rate Per 100,000 Age Standardized (https://www.worldlifeexpectancy.com/causeof-death/leukemia/by-country/female)

It is not a hereditary disease, but sometimes it can be diagnosed in representatives of several generations of the same family. In some hereditary or congenital diseases, the mechanism for repairing genetic material (DNA) is impaired, and the risk of acute leukemia is significantly increased.

The "blood cancer" is a complex disease and has many different types and subtypes. By the nature of the course of the disease, leukemia is divided into two main forms: acute and chronic. It is very difficult to find the cause of leukemia. It is known, however, that if a patient has been previously diagnosed with a different type of cancer, then this can in some cases provoke leukemia (secondary leukemia).

Risk factors:

1) "wrong lifestyle" - drinking alcoholic beverages, smoking and chewing tobacco.

2) weakening of immunity, in particular severe viral infections;

3) radiation exposure;

4) contact with chemicals;

5) chemotherapy.

The symptoms of leukemia depend on the type of leukemia and are often nonspecific. The most common signs and symptoms are as follows:

1) fever or chills;

2) tiredness, weakness;

3) frequent infections;

4) weight loss and so on.

Doctors often use a number called 5 - year survival, which refers to the percentage of patients who live at least 5 years after being diagnosed with cancer. In acute leukemia, children with no disease are likely to be completely cured after five years. This is due to the fact that after so many years, the return of this cancer is very rare. Survival rates are a convention.

The incidence of leukemia has stabilized in recent years, while in some countries there is a slight increase. A pronounced decrease in mortality is noted for childhood leukemia. Mortality from leukemia is also declining in adults, but this decline in adults is much more modest than in children. Deaths from leukemia are on the rise among the elderly. The main methods of treating the disease: chemotherapy - affecting cancer cells with chemicals; radiation therapy - affecting cancer cells with specific radiation; stem cell transplant.

\section{Literature Survey}

The T-LGL survival signaling network was constructed by adapting and simplifying a network describing the normal CTL activationAICD process.

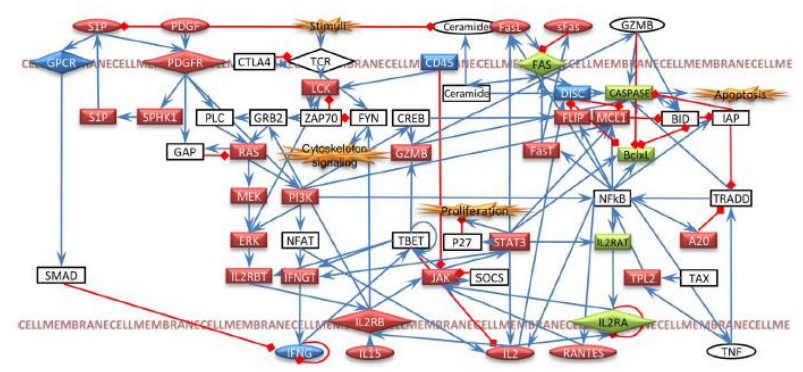

The T-LGL survival signaling network incorporates the most unique interactions through which all known deregulations in leukemic TLGL are connected, in the context of normal CTL activation and AICD signaling. Proteins, mRNAs, and small molecules (such as lipids) were represented as nodes. "Cytoskeleton signaling", "Proliferation" and "Apoptosis" were also included as nodes to summarize the biological effects of a group of components in the signaling pathways and serve as the indicators of cell fate. Because of the unknown etiology of T-LGL leukemia, was used "Stimuli" as a node to 
indicate antigen stimulation. This network contains 58 nodes and 123 edges. [2]

Differential equations describe gene expression changes as a function of the expression of other genes and environmental factors. Thus, they are adequate to model the dynamic behavior of GRNs in a more quantitative manner.[3]

Authors in work [4] give an example of realistic GRN. In this model, the cancerous states are identified with "undesired" attractors. The current state of GRN is described by the vector $X(t)=\left(x_{1}(t), \ldots, x_{n}(t)\right)$, where $t$ is time. As a disease progresses, this vector tends to a "wrong" attractor. The goal of the controllability problem is to redirect the trajectory $X(t)$ to a "normal" attractor, which in real life terms means to developacure. [4]

When mathematical modeling is used to predict the behavior of a GRN, the results need to be compared with experimental data. Developmental GRNs are built hierarchically from simple, recurring building blocks and motifs. [5]

\section{Problem Formulation}

The general form of writing the n-dimensional dynamical system, that is expected to model a genetic regulatory network, is

$$
\left\{\begin{array}{l}
x^{\prime}{ }_{1}=\frac{1}{1+e^{-\mu_{1}\left(w_{11} x_{1}+w_{12} x_{2}+\cdots+w_{1 \mathrm{n}} x_{\mathrm{n}}-\theta_{1}\right)}}-v_{1} x_{1}, \\
x^{\prime}{ }_{n}=\frac{1}{1+e^{-\mu_{\mathrm{n}}\left(w_{\mathrm{n} 1} x_{1}+w_{\mathrm{n} 2} x_{2}+\cdots+w_{\mathrm{nn}} x_{\mathrm{n}}-\theta_{\mathrm{n}}\right)}}-v_{\mathrm{n}} x_{\mathrm{n}}
\end{array}\right.
$$

where $\mu_{i}>0$ and $\theta_{i}$ are parameters, and $w_{i j}$ are elements of the $n \times n$ regulatory matrix $W$. The regulatory matrix contains information about relation between elements of a network(any element can be positive, negative, or zero, meaning activation, inhibition, or no relation).

The nullclines of system (1) are given by the equations

$$
\left\{\begin{array}{l}
x_{1}=\frac{1}{v_{1}} \cdot \frac{1}{1+e^{-\mu_{1}\left(w_{11} x_{1}+w_{12} x_{2}+\cdots+w_{1 \mathrm{n}} x_{\mathrm{n}}-\theta_{1}\right)}}, \\
x_{\mathrm{n}}=\frac{1}{v_{\mathrm{n}}} \cdot \frac{1}{1+e^{-\mu_{\mathrm{n}}\left(w_{\mathrm{n} 1} x_{1}+w_{\mathrm{n} 2} x_{2}+\cdots+w_{\mathrm{nn}} x_{\mathrm{n}}-\theta_{\mathrm{n}}\right)}}
\end{array}\right.
$$

The geometrical nullclines method is productive in the study of systems of ODE. Knowledge of nullclines allows to approximately feel the vector field, corresponding to a system of ODE. Besides, the critical points (equilibria) are the croos-points of nullclines. To follow the evolution of the vector $\mathrm{X}(\mathrm{t})$ one have to understand the architecture of a network. The main elements of this architecture are attractors of the system. Since the size of a network in the works [3], [4] is about 60 elements, one should be prepared to deal with relatively large systems. Both numerical and qualitative study of such systems is a challenging problem. In the sequel we will show how systems of arbitrary size can be constructed. We provide also the extended example of such system of order six.

\section{Three-element GRN}

Consider the three dimensional system

$$
\left\{\begin{array}{l}
x_{1}^{\prime}=\frac{1}{1+e^{-\mu_{1}\left(w_{11} x_{1}+w_{12} x_{2}+w_{13} x_{3}-\theta_{1}\right)}}-v_{1} x_{1}, \\
x_{2}^{\prime}=\frac{1}{1+e^{-\mu_{2}\left(w_{21} x_{1}+w_{22} x_{2}+w_{23} x_{3}-\theta_{2}\right)}}-v_{2} x_{2}, \\
x_{3}^{\prime}=\frac{1}{1+e^{-\mu_{3}\left(w_{31} x_{1}+w_{32} x_{2}+w_{33} x_{3}-\theta_{3}\right)}}-v_{3} x_{3} .
\end{array}\right.
$$

The first nullcline is in the set

$$
\left\{\left(x_{1}, x_{2}, x_{3}\right): 0<x_{1}<\frac{1}{v_{1}},\left(x_{2}, x_{3}\right) \in R^{2}\right\},
$$

the second nullcline is in the set

$$
\left\{\left(x_{1}, x_{2}, x_{3}\right): 0<x_{2}<\frac{1}{v_{2}},\left(x_{1}, x_{3}\right) \in R^{2}\right\},
$$

and the third one is in the set

$$
\left\{\left(x_{1}, x_{2}, x_{3}\right): 0<x_{3}<\frac{1}{v_{3}},\left(x_{1}, x_{2}\right) \in R^{2}\right\} .
$$

All critical points are located in the open parallelepiped

$$
\begin{gathered}
\left\{\left(x_{1}, x_{2}, x_{3}\right): 0<x_{1}<\frac{1}{v_{1}}, 0<x_{2}<\frac{1}{v_{2}}, 0<\right. \\
\left.x_{3}<\frac{1}{v_{3}}\right\}=: G .
\end{gathered}
$$

Due to the structure of the system and properties of sigmoidal functions, the vector field, defined by the system of ODE, is directed inward on the border of $G$. Therefore it is invariant with respect to the system. We can construct the 6-dimensional system with multiple parameters. This system will consist of two 3-dimensional systems, which can be independent and can be made coupled by introducing additional elements. 
First we consider the 3-dimensional system with the regulatory matrix

$$
W=\left(\begin{array}{ccc}
1 & 2 & 1 \\
-2 & 1 & 1 \\
0 & 0 & 1
\end{array}\right)
$$

and other parameters $\mu_{1}=\mu_{2}=\mu_{3}=7, \theta_{1}=2, \theta_{2}=0$, $\theta_{3}=0.5, v_{1}=1 ; v_{2}=1, v_{3}=1$.

For this set of data the three nullclines are located as shown in figure below.

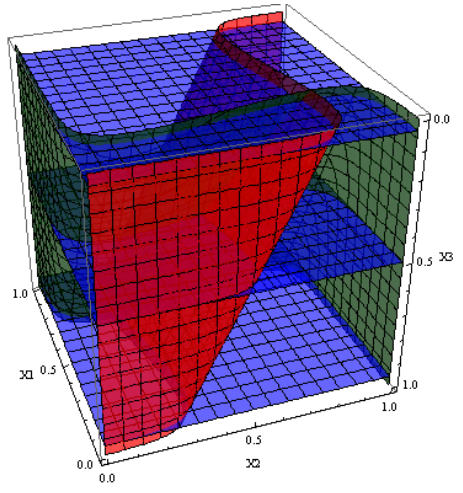

Figure 1

There are exactly three critical points, which are the cross-points of three nullclines. They are located at different $x_{3}$-heights. All of them are nonattractive critical points. Standard linearization analysis provides the characteristic numbers

$\lambda_{1}=-0.74493, \lambda_{2,3}=0.3825 \pm 2.7488 \mathrm{i}$,

$\lambda_{1}=0.4456, \quad \lambda_{2,3}=-0.443 \pm 0.6077 \mathrm{i}$,

$\lambda_{1}=-0.74492, \quad \lambda_{2,3}=0.3825 \pm 2.7488 \mathrm{i}$

for the critical points. The attractors exist however, in the form of a periodic solution. They are depicted in the below figure together with several solutions accumulating on it. This provides an example of multiple three dimensional attractors which all are not points.

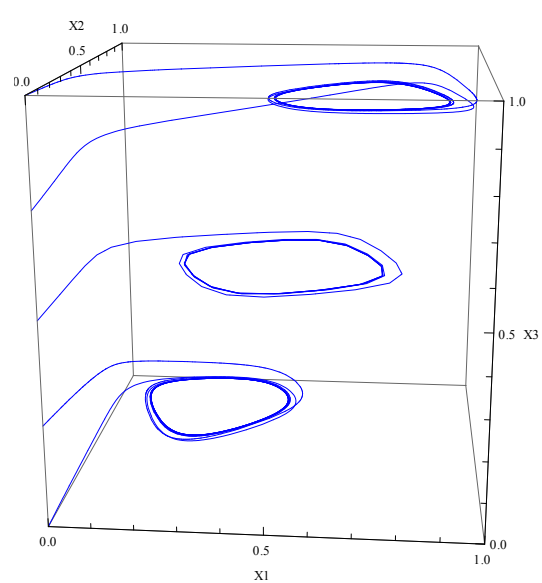

Figure 2: Periodic solutions in 3D

To see, that solutions tend to a periodic attractor, the graphs of all components of a solution versus time $t$ are provided in Figure 3. One of the components is eventually constant, so the attractor is $x_{3}$-"flat".

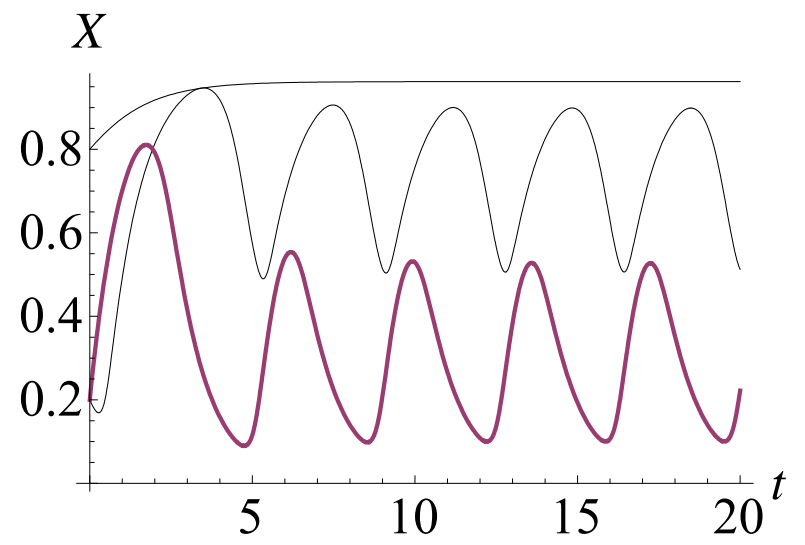

Figure 3: Solutions $x_{1}(t) ; x_{2}(t) ; x_{3}(t)$ tending to the $x_{3}$-upper critical point.

\section{Six-element GRN}

Consider the 6-dimensional system

$$
\left\{\begin{array}{c}
x^{\prime}{ }_{1}=\frac{1}{1+e^{-\mu_{1}\left(w_{11} x_{1}+w_{12} x_{2}+w_{13} x_{3}-\theta_{1}\right)}}-v_{1} x_{1}, \\
x^{\prime}{ }_{2}=\frac{1}{1+e^{-\mu_{2}\left(w_{21} x_{1}+w_{22} x_{2}+w_{23} x_{3}-\theta_{2}\right)}}-v_{2} x_{2}, \\
x^{\prime}{ }_{3}=\frac{1}{1+e^{-\mu_{3}\left(w_{33} x_{3}-\theta_{3}\right)}}-v_{3} x_{3} .
\end{array}\right.
$$




$$
\left\{\begin{array}{c}
x^{\prime}{ }_{4}=\frac{1}{1+e^{-\mu_{4}\left(w_{44} x_{4}+w_{45} x_{4}+w_{46} x_{4}-\theta_{4}\right)}}-v_{4} x_{4}, \\
x^{\prime}{ }_{5}=\frac{1}{1+e^{-\mu_{5}\left(w_{54} x_{5}+w_{55} x_{5}+w_{56} x_{5}-\theta_{5}\right)}}-v_{5} x_{5}, \\
x^{\prime}{ }_{6}=\frac{1}{1+e^{-\mu_{6}\left(w_{66} x_{6}-\theta_{6}\right)}}-v_{6} x_{6},
\end{array}\right.
$$

which consists of two uncoupled 3-dimensional systems. Let all the parameters in $3 \mathrm{D}$ systems be as in the system considered in the previous section. Then the six-dimensional system has several attractors which are the products of two three-dimensional periodic trajectories.

Projections of this attractor on three-dimensional subspaces are in figures 4-6 below. The solution of the system (2) with the initial conditions $x_{1}(0)=x_{2}(0)=x_{4}(0)=x_{5}(0)=0, \quad x_{3}(0)=0.8$, $x_{6}(0)=0.2$

is depicted.

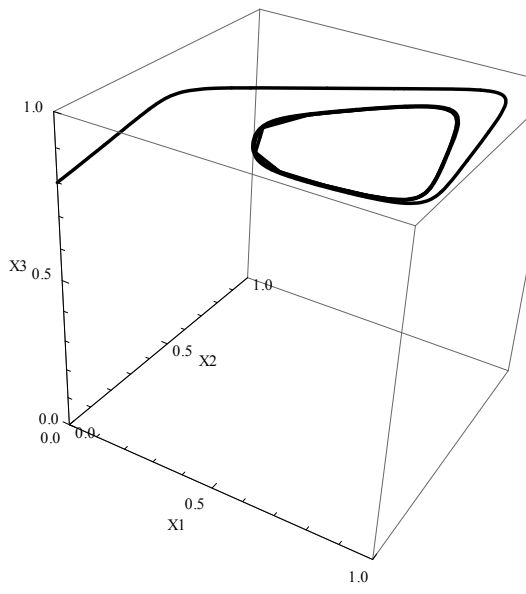

Figure 4: projection $\left(x_{1}, x_{2}, x_{3}\right)$.

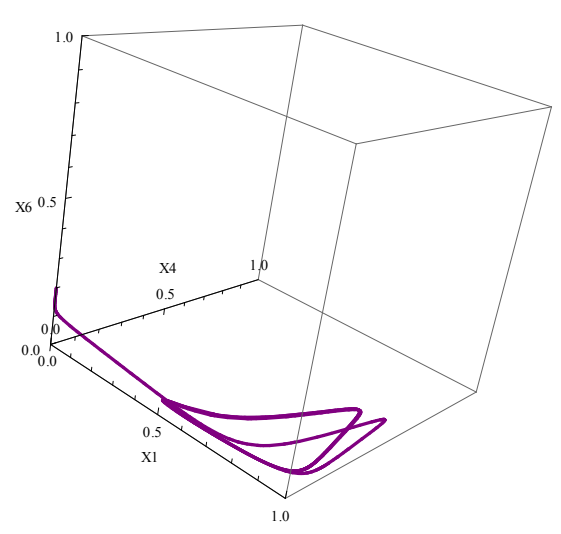

Figure 5: projection $\left(x_{1}, x_{4}, x_{6}\right)$.

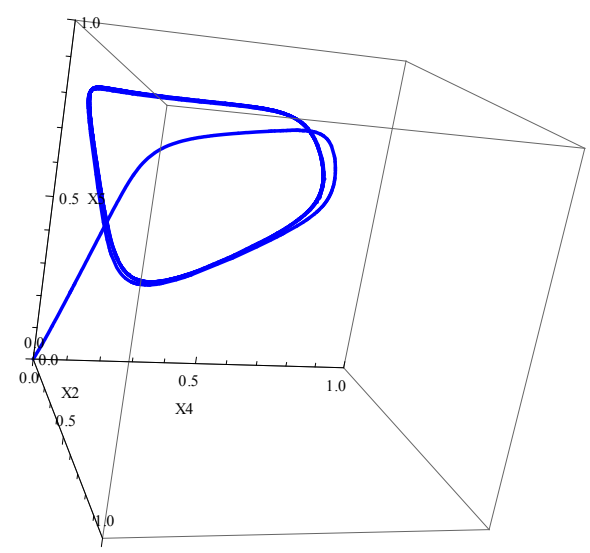

Figure 6: projection $\left(x_{2}, x_{4}, x_{5}\right)$.

\section{Six element coupled system}

Consider perturbed 6-dimensional system with the regulatory matrix

$$
W=\left(\begin{array}{cccccc}
1 & 2 & 1 & 0 & 0 & 0 \\
-2 & 1 & 1 & 0 & 0 & 0 \\
0 & 0 & 1 & -0.5 & 0 & 0 \\
0 & 0 & 0.8 & 1 & 2 & 1 \\
0 & 0 & 0 & -2 & 1 & 1 \\
0 & 0 & 0 & 0 & 0 & 1
\end{array}\right)
$$

Projections of this attractor on three-dimensional subspaces are in figures 7-9 below. The initial conditions are taken the same as for unperturbed system above. 


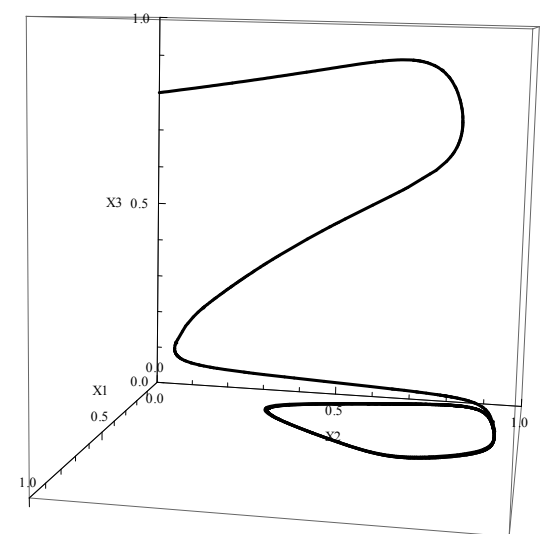

Figure 7: projection $\left(x_{1}, x_{2}, x_{3}\right)$.

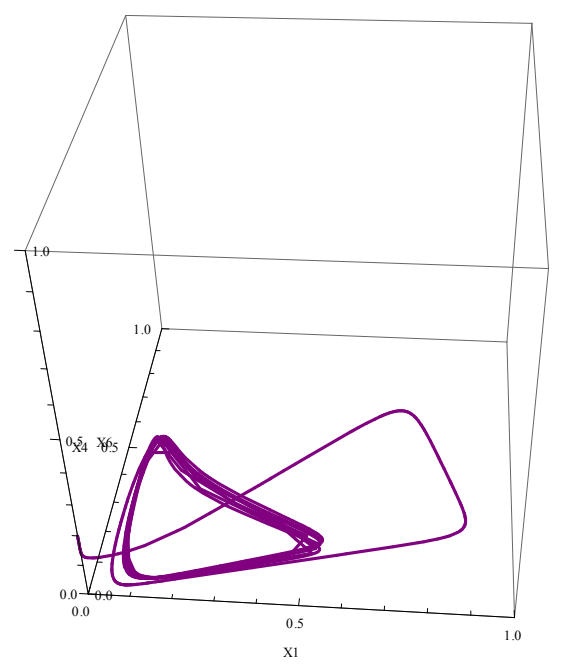

Figure 8: projection $\left(x_{1}, x_{4}, x_{6}\right)$.

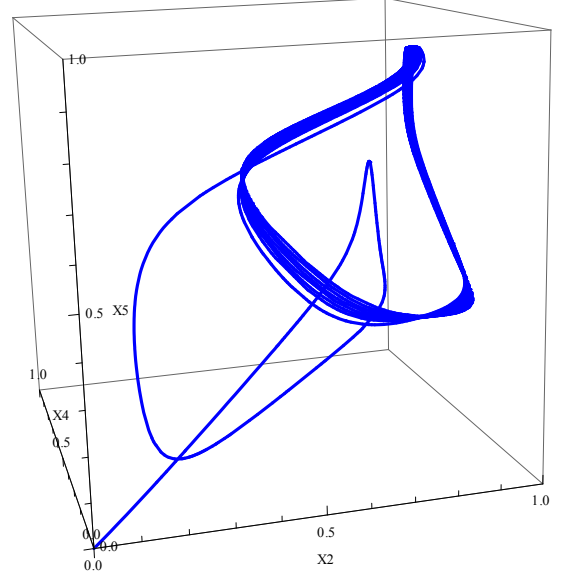

Figure 9: projection $\left(x_{2}, x_{4}, x_{5}\right)$.

Solutions of the perturbed system tends to the periodic attractor as shown in Figure 10.

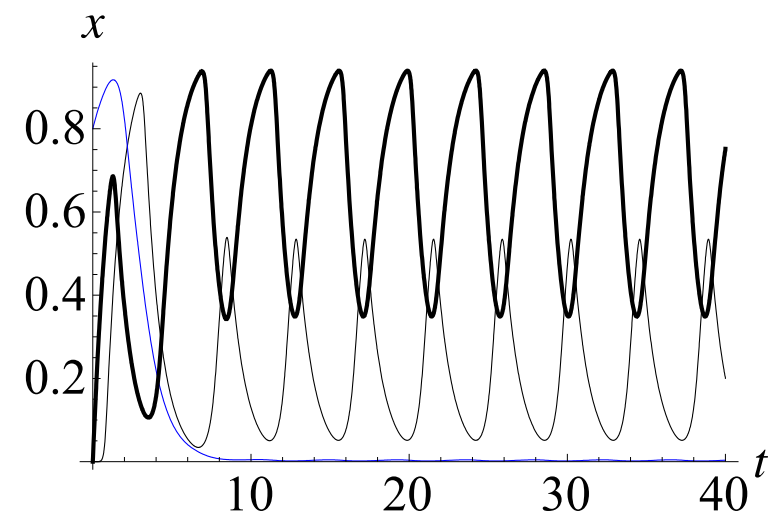

Figure 10: Solutions $x_{1}(t) ; x_{3}(t) ; x_{5}(t)$.

Three-dimensional systems can have stable critical points and attractive periodic solutions. Six-dimensional systems can be constructed which possess attractors of periodic type. Solutions tend to periodic attractors, becoming periodic in indefinite time. Perturbed sixdimensional systems exist, which have an attractor of the periodic type. Periodic attractors can coexist with stable equilibria.

\section{Conclusion}

The problem of studying genomes and principles of their functioning is one of the most challenging in nowadays biology. These studies require obtaining huge amount of experimental data, storing and processing them. Elsewhere banks of genomic data are founded and large institutes deal with analysing them. Mathematical modelling introduces a kind of order in a big amount of data.

Knowledge of principles and in any practical case details of specific gene networks is needed for successful leukemia treatment.

Our aim is to investigate high-dimensional systems such that were considered in the article [6]: 60-dimensional system for leukemia treatment, to find attractors and to find understanding how to redirect the trajectory from "wrong" (leukemia disease) to a "normal" attractor (leukemia treatment).

More on related problems can be found in the articles and books [7]-[21].

ESF Project No. 8.2.2.0/20/I/003 "Strengthening of Professional Competence of Daugavpils University Academic Personnel of Strategic Specialization Branches 3rd Call" 


\section{References:}

[1] https://www.worldlifeexpectancy.com/cause-ofdeath/leukemia/by-country

[2] Zhang R, Shah MV, Yang J, et al. Network model of survival signaling in large granular lymphocyte leukemia. Proc Natl Acad Sci $U$ S A.2008;105(42):16308-16313.

doi:10.1073/pnas.0806447105

[3] Hecker M, Lambeck S, Toepfer S, van Someren E, Guthke R. Gene regulatory network inference: data integration in dynamic models-a review. Biosystems. $2009 \quad$ Apr;96(1):86-103.doi: 10.1016/j.biosystems.2008.12.004. Epub 2008 Dec 27. PMID: 19150482.

[4] Felix Sadyrbaev, Inna Samuilik, Valentin Sengileyev, "On Modelling of Genetic Regulatory Networks," WSEAS Transactions on Electronics, vol. 12, pp. 73-80, 2021

[5] William J.R. Longabaugh, Eric H. Davidson, Hamid Bolouri, Computational representation of developmental genetic regulatory networks, Developmental Biology, Volume 283, Issue 1, 2005, Pages 1-16, ISSN 00121606,https://doi.org/10.1016/j.ydbio.2005.04.023.

[6] Le-Zhi Wang, Ri-Qi Su,Zi-Gang Huang, Xiao Wang, Wen-Xu Wang, Celso Grebogi and YingChengLai, A geometrical approach to control and controllability of nonlinear dy-namical networks. Nature Communications, Volume7,Articlenumber:11323(2016), DOI:10.1038/ncomms11323

[7] H. D. Jong. Modeling and Simulation of GeneticRegulatory Systems: A Literature Re- view, J. Comput Biol. 2002;9(1):67-103, DOI: $10.1089 / 10665270252833208$

[8] T.Schlitt.ApproachestoModelingGeneRegulatoryNet works:AGentleIntroduc-tion.In:Silico Systems Biology.

MethodsinMolecularBiology(MethodsandProtocols),HumanaPress, vol.1021(2013),1335,doi:10.1007/978-1-62703-450-0

[9] A. Spirov and D. Holloway. Using evolutionarycomputationstounderstandthedesignand evolution of gene and cell regulatory net-works. Methods. 2013 July 15; 62(1):3955.

[10]Y. Koizumi et al. Adaptive Virtual NetworkTopology Control Based on Attractor Selec-tion.Journal of Lightwave Technology(ISSN: 0733-8724), Vol.28 (06/2010), Issue

11 ,

pp.1720-

\section{DOI:10.1109/JLT.2010.2048412}

[11]H. D. Jong. Modeling and Simulation of GeneticRegulatory Systems: A Literature Re- view, J. Comput Biol. 2002;9(1):67-103, DOI: $10.1089 / 10665270252833208$

[12]S. Atslega, F. Sadyrbaev, I. Samuilik. On Modelling Of Complex Networks. Engineering for Rural Development(ISSN 1691-5976), 2021, pp. 10091014. http://tf.llu.lv/conference/proceedings2021/Papers/TF 223.pdf

[13]V.W.Noonburg.Differential Equations:From Calculus to Dynamical Systems, Providence, Rhode Island:MAA Press, 2019, $2^{\text {nd }}$ edition.

[14]Felix Sadyrbaev, Inna Samuilik, Valentin Sengileyev, "On Modelling of Genetic Regulatory Networks," WSEAS Transactions on Electronics, vol. 12, pp. 73 80,2021

[15]F.Sadyrbaev. Planar differential systems arising in network regulation theory. AdvancedMathematicalModels\&Applications, Vol.4,No.1,2019,pp.70-78

[16]S.Atslega,D.Finaskins,F.Sadyrbaev.On a Planar Dynamical System Arising in the Network Control Theory. Mathematical Modelling and Analysis, 21 (2016), N 3, 385 -398.

[17]A.Das, A.B.Roy, PrithaDas. Chaos ina three dimensional neural network. AppliedMathematicalModelling24(2000)511-522

[18] WeimingYe,XiaodongHuang,XuhuiHuang, PengfeiLi, Qinzhi Xia, GangHu. Selfsustained oscillationsof complex genomic regulatory networks.Physics LettersA374(2010)25212526.doi:10.1016/j.physlet a.2010.04.015

[19]R.Edwards and L. Ironi. Periodic solutions of gene networks with steep sigmoidal regula-tory functions. Physica D, 282 (2014), 1 15.https://doi.org/10.1016/j.physd.2014.04.013

[20]E. Brokan and F. Sadyrbaev, Attrac-tioninndimensionaldifferentialsystemsfromnetworkregulationt heory,Math-ematicalMethodsinthe Applied Sciences,41,2018,Issue 17,7498-

7509https://doi.org/10.1002/mma.5086

[21]C. Furusawa, K. Kaneko. A generic mech-anism for adaptive growth rate regulation.PLoSComput Biol 4(2008), 1, e3. 00350042. https://doi.org/10.1371/journal.pcbi.0040003. 


\section{Contribution of individual}

authors to the creation of a scientific article (ghost writing policy)

All authors have contributed equally to creation of this article.

Sources of funding for research presented in a scientific article or scientific article itself

There is no funding for this article.

\section{Creative Commons Attribution}

\section{License 4.0 (Attribution 4.0}

\section{International, CC BY 4.0)}

This article is published under the terms of the

Creative Commons Attribution License 4.0

https://creativecommons.org/licenses/by/4.0/deed.e

n_US 